\title{
Functional interaction of PkcA and PldB regulate aggregation and development in Dictyostelium discoideum
}

\author{
Sean Singh ${ }^{\text {a }}$, Wasima Mohamed ${ }^{\mathrm{b}}$, Annelie Aguessy ${ }^{\mathrm{a}}$, Ella Dyett ${ }^{\mathrm{a}, 1}$, Shriraj Shah ${ }^{\mathrm{a}, 1}$, Mohammedasad Khan ${ }^{\mathrm{a}, 1}$, \\ Ramamurthy Baskar ${ }^{\mathrm{b}}$, Derrick Brazill ${ }^{\mathrm{a}, *}$ \\ a Department of Biological Sciences, Hunter College and The Graduate Center, The City University of New York, New York, NY, USA \\ ${ }^{\mathrm{b}}$ Department of Biotechnology, Bhupat and Jyoti Mehta School of Biosciences, Indian Institute of Technology-Madras, Chennai 600036, India
}

\section{A R T I C L E I N F O}

\section{Article history:}

Received 12 January 2017

Received in revised form 24 February 2017

Accepted 24 February 2017

Available online 28 February 2017

\section{Keywords:}

Dictyostelium

Development

Quorum sensing

Actin cytoskeleton

PKC

PLD

\begin{abstract}
A B S T R A C T
Multicellular development in Dictyostelium discoideum involves tightly regulated signaling events controlling the entry into development, initiation of aggregation and chemotaxis, and cellular differentiation. Here we show that PkcA, a Dictyostelium discoideum Protein Kinase C-orthologue, is involved in quorum sensing and the initiation of development, as well as cAMP sensing during chemotaxis. Additionally, by epistasis analysis we provide evidence that PkcA and PldB (a Phospholipase D-orthologue) functionally interact to regulate aggregation, differentiation, and cell-cell adhesion during development. Finally, we show that PkcA acts as a positive regulator of intracellular PLD-activity during development. Taken together, our results suggest that PkcA act through PldB, by regulating PLD-activity, in order to control events during development.
\end{abstract}

(c) 2017 Elsevier Inc. All rights reserved.

\section{Introduction}

Dictyostelium discoideum is a popular model for studying development, as well as nutrient sensing, cytoskeletal regulation, and chemotaxis. This is due to its relative simplicity in comparison to higher order systems, while simultaneously possessing highly conserved signaling pathways, particularly during its development. Dictyostelium discoideum are social amoeba, which exist as individual cells under normal conditions. They live in the soil, feeding on bacteria and yeasts until their food sources are depleted. At this point, they begin a developmental program in which, they shift from a unicellular lifestyle to a multicellular one over the course of approximately $24 \mathrm{~h}[1,2]$. Starving cells secrete cyclic adenosine monophosphate (cAMP), which serves as a chemoattractant to other starving cells. As cells chemotax towards the source of cAMP, they reorganize their cytoskeleton and polarize along the cAMP gradient. This results in cells coming together, forming a mound of cells that differentiate into prestalk or prespore populations. Cells within the mound self-segregate eventually differentiating into stalk or spore cells that make up the final fruiting body [3].

Abbreviations: cAMP, cyclic adenosine monophosphate; CMF, Conditioned Media Factor; PA, phosphatidic acid; DAG, diacylglycerol; PKC, protein kinase C; PLD, phospholipase D.

* Corresponding author at: Dept. of Biological Sciences, Hunter College, 695 Park Ave, New York, NY 10065-5085, USA.

E-mail address: brazill@genectr.hunter.cuny.edu (D. Brazill).

1 These authors contributed equally.
Entry into the developmental program is tightly regulated at the individual and population levels [4]. The chemotactic response to cAMP, as well as developmental competency, is regulated by the levels of a secreted protein, Conditioned Medium Factor (CMF) [5]. Individual cells will secrete both CMF and cAMP at the onset of starvation. CMF acts as a quorum sensing factor, the greater the number of starving cells present, the higher the level of CMF [6]. When CMF concentrations are high, cells become responsive to cAMP and aggregate. Quorum sensing ensures that fruiting body formation will not begin until a critical number of cells are starving to produce a viable fruiting body [7].

CMF sensing and the CAMP response are linked to each other through the activity of pldB, one of the Dictyostelium discoideum orthologues of PLD1, a mammalian phospholipase D [8]. pldB serves as a negative regulator of aggregation and development, by regulating quorum sensing, motility, and F-actin organization [8-10]. These regulatory effects are thought to be mediated by its enzymatic activity, producing phosphatidic acid (PA) from phosphatidylcholine. Activation of CMF signaling, due to high numbers of starving cells, downregulates PldB activity, allowing cAMP signaling to occur [8]. The downregulation of PldB involves the CMF-mediated activation of PLC, a Dictyostelium discoideum orthologue of phospholipase $C[5,8,11,12]$. This may occur in part through the PLC-mediated production of the phospholipid secondary signaling molecule diacylglycerol (DAG). DAG production is developmentally regulated, particularly during differentiation, and culmination [13]. However, no direct link between PLC and PldB has been identified. 
One possible link between PLC and PldB is a putative PKC orthologue, $p k c A$, which was recently described in Dictyostelium discoideum. pkcA is expressed at the same time as PldB, and regulates many developmental processes including cAMP relay, cellular differentiation, and cell-cell adhesion [14]. The Protein Kinase C (PKC)-class of kinases are an important family of Ser/Thr-kinases within animals and fungi. There are at least ten different PKC isoforms in higher organisms, which are classified by their responsiveness to the secondary signaling molecules diacyclglycerol and/or $\mathrm{Ca}^{2+}$ [15]. Different PKC isoforms have been shown to be involved in the regulation of many critical signaling processes, such as those regulating the cell cycle, cell growth and proliferation, nutrient sensing, development, cell density sensing, cytoskeletal organization, cell motility, and cellular differentiation during development [15-24]. Misregulation of PKC-mediated signaling in these processes has been implicated in many diseases, including developmental defects, metabolic disorders, and various cancers [16-19,23-26].

One of the most well characterized signaling interactions of PKC is its interactions with both phospholipase C (PLC) and phospholipase D (PLD). PLC serves as the major producer of diacylglycerol in the cell, which can act as a powerful activator of PKC. Activated PKC then regulates PLD-activity, thus controlling numerous downstream signaling pathways [27-29]. In Dictyostelium discoideum, the presence of an activated PLC upstream of PldB provides a source of DAG [5], potentially activating PkcA during development. This sequence of signaling events would recapitulate the PLC-PKC-PLD pathway [29], regulating other downstream signaling pathways during development. Here we further investigate the role of PkcA during quorum sensing and aggregation using different genetic techniques. Additionally, we establish a functional relationship between PkcA and PldB in a number of developmentally relevant processes.

\section{Materials and methods}

\subsection{Strains \& cell culture}

Dictyostelium discoideum wild type cells, Ax2, were grown axenically in HL5 medium including Glucose (Formedium ${ }^{\mathrm{TM}}$, Norfolk, UK) at $22^{\circ} \mathrm{C}$ in shaking suspension culture. To generate cells lacking PkcA, nucleotides 2198-3480 of the $p k c A$ gene were replaced with the blasticidin resistance cassette from pBsr519 [30]. This construct was ligated into the pCR2.1®-TOPO ${ }^{\circledR}$ TA vector (Invitrogen, Carlsbad, CA). For transformation, the vector was linearized using NotI, and transformed into Ax2 cells via electroporation [31]. Transformants were selected using $10 \mu \mathrm{g} / \mathrm{mL}$ blasticidin. $p k c A^{-}$, and $p l d B^{-}$[9] cells were grown in HL5 supplemented with $10 \mu \mathrm{g} / \mathrm{mL}$ blasticidin. pkcA overexpressing cells ( $p k c A O E$ ) [14] were grown in HL5 supplemented with $20 \mu \mathrm{g} / \mathrm{mL}$ G418. pldB overexpressing cells ( $p l d B O E$ ) [9] cells were grown in HL5 supplemented with $6 \mu \mathrm{g} / \mathrm{mL}$ G418. $p k c A^{-} / p l d B O E$ cells were made by transforming $p k c A^{-}$cells via electroporation with the previously described pldB-overexpression construct, and grown in HL5 supplemented with $10 \mu \mathrm{g} / \mathrm{mL}$ blasticidin and $20 \mu \mathrm{g} / \mathrm{mL}$ G418. For development, log phase cells $\left(1.0-5.0 \times 10^{6}\right.$ cells $\left./ \mathrm{mL}\right)$ were collected and washed twice in PBM $\left(20 \mathrm{mM} \mathrm{KH}_{2} \mathrm{PO}_{4}, 0.01 \mathrm{mM} \mathrm{CaCl}_{2}, 1 \mathrm{mM} \mathrm{MgCl}_{2}\right.$, pH 6.1 by $\mathrm{KOH}$ ), then plated at a density of $1.0 \times 10^{7}$ cells $/ \mathrm{mL}$ on agar, or nitrocellulose filter pads (EMD Millipore, Billerica, MA) over an area of approximately $1 \mathrm{~cm}^{2}$ and allowed to develop for various amounts of time at $22{ }^{\circ} \mathrm{C}[32]$.

\subsection{Low cell-density assay}

Log phase cells were collected, washed twice in PBM, then starved for $24 \mathrm{~h}$ under submerged monolayer conditions over a surface area of $2 \mathrm{~cm}^{2}$ at densities of $224 \times 10^{3}, 112 \times 10^{3}, 56 \times 10^{3}, 28 \times 10^{3}$, $14 \times 10^{3}$, and $7 \times 10^{3}$ cells $/ \mathrm{cm}^{2}$ in $400 \mu \mathrm{L}$ PBM, or PBM supplemented with either $50 \mu \mathrm{M}$ 1,2-dioctanoyl-sn-glycero-3-phosphate (PA)(Avanti Polar Lipids Inc., Alabaster, AL), or $0.1 \%$ butanol.

\subsection{Chimeras and spore formation rescue assay}

$1.0 \times 10^{7} \log$ phase cells were collected and washed in PBM. Chimera were created by mixing $10 \% \mathrm{Ax} 2$ cells with $90 \%$ of either $p k c A^{-}$, pldBOE, or $p k c A^{-} / p l d B O E$ cells, plating the mixture on a nitrocellulose filter pad at a density of $1.0 \times 10^{7}$ cells $/ \mathrm{mL}$ and allowing the mixtures to develop for $36 \mathrm{~h}$. Spores were harvested in $1 \mathrm{~mL}$ buffer (PBM with $10 \mathrm{mM}$ EDTA, $0.1 \%$ Nonidet P-40 detergent) and counted on a hemocytometer to determine the number of spores collected. Spore yields from chimera were normalized to those of fruiting bodies comprised of only Ax2 cells.

\subsection{Cell-cell cohesion assay}

Cell-cell cohesion assay was performed as previously described [33], with some modification. Log phase cells were collected and washed three times in PBM, then resuspended in PBM to a final density of $2.0 \times 10^{7}$ cells $/ \mathrm{mL}$. Two milliliters of cell suspension was collected, transferred to a $50-\mathrm{mL}$ conical tube and shaken horizontally (175 rpm) on a rotary platform shaker for $3 \mathrm{~h}$ at $22^{\circ} \mathrm{C}$. $2.5 \times 10^{6}$ cells were collected and diluted to $1 \mathrm{~mL}$ final volume, then vortexed vigorously to disperse aggregates. Cells were shaken slowly at $140 \mathrm{rpm}$ for $40 \mathrm{~min}$, to allow aggregates to reform, then single and duplex cells were counted on a hemocytometer.

\subsection{Cell-substrate adhesion assay}

Cell-substrate adhesion assay was performed as previously described [33]. Log phase cells were collected and washed three times in PBM, then resuspended in PBM to a final density of $1.0 \times 10^{6}$ cells $/ \mathrm{mL}$. Four milliliters of cell suspension was placed in a $50-\mathrm{mL}$ glass cell culture flask and shaken $(120 \mathrm{rpm})$ on a rotary platform shaker for $10 \mathrm{~min}$. The cells were then incubated without shaking for $2 \mathrm{~h}$ to allow them to adhere. The flasks were then gently shaken at $60 \mathrm{rpm}$ for $3 \mathrm{~min}$, and the supernatants collected. Non-adherent cells were counted on a hemocytometer.

\subsection{Actin staining}

Log phase cells were collected and washed twice in PBM, then resuspended in PBM to a density of $1.5 \times 10^{6}$ cells $/ \mathrm{mL}$. Cells were seeded on an 8-well chambered coverglass (Thermo Fisher Scientific, Waltham, MA) at a density of $2.0 \times 10^{5}$ cells/well and allowed to starve for $6 \mathrm{~h}$. Cells were then fixed in 3.7\% formaldehyde in PBSN (PBS + 0.05\% NP40) for $10 \mathrm{~min}$ at room temperature. Slides were then washed in PBSN $+16.7 \mathrm{mM}$ glycine for $5 \mathrm{~min}$ at room temperature, followed by PBSN for $15 \mathrm{~min}$ at room temperature. Cells were stained with Alexa Fluor 546-conjugated phalloidin (Molecular Probes, Eugene, OR) at $8 \mathrm{U} / \mathrm{mL}$ in PBSN for $1 \mathrm{~h}$ in the dark at room temperature. Cells were then washed twice in PBSN. SlowFade Gold (Molecular Probes, Eugene, OR) mounting reagent was added before adding a coverslip and sealing. Images were taken with a Leica confocal microscope using the $63 \times$ objective. Cell circularity and roundness were measured using ImageJ software (NIH, Bethesda, MD), where circularity is defined as $\frac{4 \pi(a r e a)}{\text { perimeter }^{2}}$ and roundness is defined as length of minor axis.

\subsection{Nuclei staining}

Log phase cells were collected and seeded on an 8-well chambered coverglass (Thermo Fisher Scientific, Waltham, MA) at a density of $1.0 \times 10^{5}$ cells/well. Cells were fixed in 95\% ethanol at room temperature for $20 \mathrm{~min}$, and allowed to air dry. Nuclei were stained using NucBlue Fixed Cell ReadyProbes Reagent (Molecular Probes, Eugene, OR) using the manufacturer's protocol. SlowFade Gold mounting reagent was added before placing the coverslip. Images were taken with a Nikon Eclipse Ti microscope using the $40 \times$ objective. 


\subsection{Phospholipase D activity assay}

Log phase cells were collected and washed twice in PBM. Cells were starved for $6 \mathrm{~h}$ on nitrocellulose filter pads at a density of $1.0 \times 10^{7}$ cells $/ \mathrm{mL}$. After $6 \mathrm{~h}$, cells were collected and Phospholipase D activity was determined utilizing a Phospholipase D Activity Colorimetric Assay Kit (BioVision Inc., Milpitas, CA) following the manufacturer's protocol.

\section{Results}

\section{1. $p k c A$ is a negative regulator of aggregation}

PkcA regulates tip dominance in late-stage aggregates and loss of $p k c A$ leads to fragmented aggregates that formed smaller fruiting bodies than wild-type [14]. However, $p k c A$ is expressed as early as $4 \mathrm{~h}$ after starvation, suggesting it serves other functions during early development as well, possibly during the initiation of aggregation. To determine the role of PkcA during early development, we examined aggregation at a low cell density. When starved under submerged culture conditions, wild-type cells aggregated at densities as low as $28 \times 10^{3} \mathrm{cells} / \mathrm{cm}^{2}$. $p k c A^{-}$cells aggregated even at densities as low as $7 \times 10^{3}$ cells $/ \mathrm{cm}^{2}$, behaving as though the CMF pathway is constitutively active. Conversely, pkcAOE cells did not aggregate at the cell densities we tested, behaving as though the CMF pathway is blocked (Table 1). This suggests that PkcA acts as a negative regulator of aggregation, potentially acting through CMF pathway.

\section{2. pkcA and pldB cooperatively regulate aggregation}

The mutants in $p k c A$ behave similarly to the previously described mutants in $p l d B$, that has acts as a negative regulator of aggregation and development (Table 1) [9]. The similarities in the aggregation phenotypes of their respective mutants suggest that $p k c A$ and $p l d B$ both work within the CMF pathway to regulate development. To assess the potential epistatic interactions during aggregation, a double mutant lacking PkcA and overexpressing PldB ( $\left.p k c A^{-} / p l d B O E\right)$ was generated. At low cell densities, the $p k c A^{-} / p l d B O E$ cells do not aggregate at densities tested, phenocopying the previously established pldBOE parent phenotype (Table 1) [9]. This suggests that PkcA and PIdB interact functionally, at least during aggregation and that PldB functions downstream of PkcA.

\section{3. pkcA and pldB cooperatively regulate development}

To determine how PkcA and PldB interact during development, we examined the developmental phenotypes of wild-type cells, $p k c A^{-}$ cells, and pkcAOE cells starved for $24 \mathrm{~h}$ on agar (Fig. 1). Wild-type cells proceeded through development and formed fruiting bodies with a normal morphology. At $24 \mathrm{~h}$, the $p k c A^{-}$cells formed very small mounds, but required $>48 \mathrm{~h}$ to fully complete development. These fruiting bodies

Table 1

Low-cell density aggregation of $p k c A$, and pldB mutant cells.

Cells were starved in PBM at the indicated cell densities in submerged monolayer culture. The field of cells was assessed for aggregation (+) or non-aggregation (-) after $24 \mathrm{~h}$, using an inverted microscope. Partial or substandard aggregation (defined as where cells are migrating towards an aggregative center, but have not yet coalesced into an aggregate) is represented as \pm . These are representative of at least 3 independent assays.

\begin{tabular}{|c|c|c|c|c|c|c|}
\hline \multirow[b]{2}{*}{ Cell type } & \multicolumn{6}{|c|}{ Presence of aggregates at cell density $\left(10^{3}\right.$ cells $\left./ \mathrm{cm}^{2}\right)$ of: } \\
\hline & 224 & 112 & 56 & 28 & 14 & 7 \\
\hline $\mathrm{Ax} 2$ & + & + & + & \pm & - & - \\
\hline$p k c A^{-}$ & + & + & + & + & + & \pm \\
\hline pkcAOE & - & - & - & - & - & - \\
\hline pldB $B^{-}$ & + & + & + & + & + & + \\
\hline pldBOE & - & - & - & - & - & - \\
\hline $\mathrm{pkcA}^{-} / \mathrm{pldBOE}$ & - & - & - & - & - & - \\
\hline
\end{tabular}

were smaller than wild-type, with enlarged sori. This suggests that PkcA is not strictly required for development, and that expression of PkcA is necessary for proper culmination and developmental timing, as well as proper cell sorting and differentiation. The pkcAOE cells were unable to aggregate, and did not undergo development, suggesting a role as a negative regulator of aggregation. When $p k c A^{-} /$pldBOE mutant cells were starved, they did not aggregate. This once again emulates the pldBOE phenotype, suggesting that $p l d B$ acts downstream of $p k c A$ in controlling development.

\section{4. pkcA and pldB cooperatively regulate cell sorting and differentiation}

$p k c A$ regulates the expression of the prestalk marker ecmA, but is expressed in prespore cells late in development [14], suggesting a role in regulating differentiation during development. $p k c A^{-}$cells have a mix of cell autonomous and non-cell autonomous defects in developmentally regulated processes. Non-cell autonomous mutations affect a cell's ability to produce a signal, which can be rescued by the presence of wild-type cells. Cell autonomous mutations are due to an internal defect in relaying a signal, which cannot be restored by the wild-type cells. The altered morphology of $p k c A^{-}$fruiting bodies suggests a defect in their ability to differentiate and form spore or stalk cells in correct proportions. To determine whether the presence of wild-type cells could restore normal spore production, we generated chimeras by mixing wild-type and mutant cells at a ratio of $10 \%$ Ax 2 cells to $90 \%$ mutant cells and determined the total spore yield of the chimeras (Fig. 2). Chimera producing $>10 \%$ spore yield when compared to fruiting bodies comprised of $\mathrm{Ax} 2$ alone were considered a rescue of spore formation phenotype while chimera producing $\leq 10 \%$ spore yield when compared to control spores were not considered to be rescued by the presence of the wild-type cells. Chimera of wild type and $p k c A^{-}$cells completed development within $36 \mathrm{~h}$, and yielded $\sim 26 \%$ spores compared to the spore yield of control fruiting bodies comprised of Ax2 cells alone. This is in excess of $10 \%$ spore yield expected of the $10 \%$ wild-type cells present in the chimera, but less than a full rescue of spore formation. This suggests that $p k c$ A plays a non-cell autonomous role in regulating spore formation, possibly regulating the proportions of spore cells to stalk cells.

In order to determine if there is an epistatic interaction between $p k c A$ and $p l d B$ during spore formation, we also generated chimeras comprised of wild-type cells and either pldBOE or $p k A^{-} / p l d B O E$ mutant cells (Fig. 2). In both cases, the presence of wild-type cells was unable to rescue spore formation with either mutant cell line. pldBOE cells produced only $\sim 4 \%$ as many spores as wild-type-only controls, while $\mathrm{pkcA}^{-}$/ pldBOE produced only $\sim 6 \%$ as many spores. Overexpression of pldB produces a cell autonomous defect during spore formation, as they were unable to be rescued by the presence of wild-type cells. Since the $p k c A^{-} / p l d B O E$ shared the phenotype of pldBOE cells, it suggests that PldB operates downstream of PkcA.

\section{5. pldB overexpression rescues adhesion defects due to loss of $p k c A$}

Multicellular development in Dictyostelium discoideum requires proper sorting within the mound, which is highly dependent on both cell-cell cohesion and cell-substrate adhesion [3]. It has previously been reported that $\mathrm{pkc} \mathrm{A}^{-}$cells exhibit decreased cell-cell cohesion compared to wild-type cells [14]. Given the apparent developmental defects present in both parental mutants, $p k c A^{-}$, and pldBOE and the $p k c A^{-}$/ pldBOE, we examined the cell-cell cohesion properties of the $\mathrm{pkcA}^{-}$/ pldBOE to determine whether $p k c A$ and pldB interact to regulate cellcell cohesion (Fig. 3A). $p k c A^{-}$cells exhibited significantly reduced cellcell cohesion while pldBOE cells did not differ significantly from wildtype cells. Additionally, we found that cell-cell cohesion in $\mathrm{pkcA}^{-}$/ pldBOE cells also did not significantly differ from wild-type cells, despite lacking PkcA. The overexpression of pldB compensated for the loss of $p k c A$ and restored proper cell-cell cohesion. This suggests a functional 


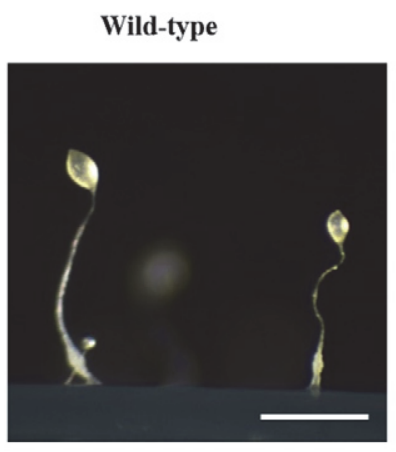

pldBOE

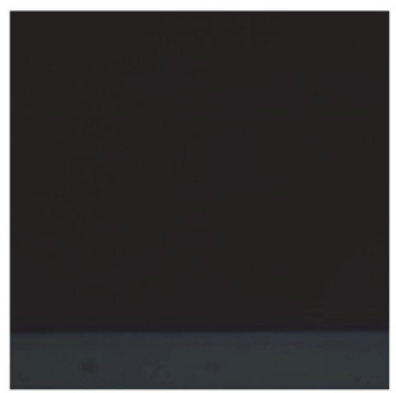

$p k c A^{-}$

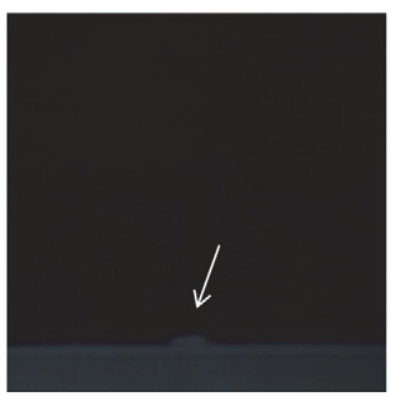

pkcA-/pldBOE

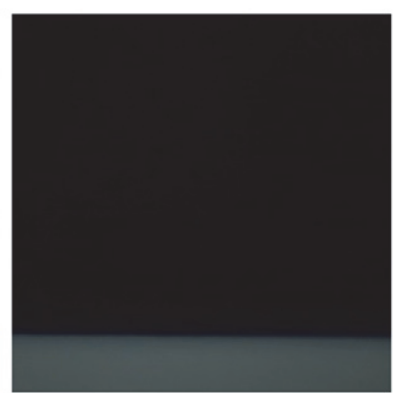

pkcAOE

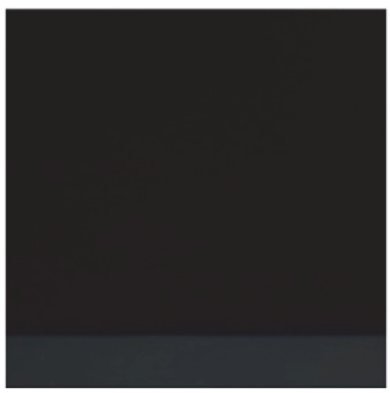

Fig. 1. Fruiting bodies on agar. Wild-type Ax2 cells, $p k c A^{-}$cells, $p k c A O E$ cells, pldBOE cells, and $p k c A^{-} / p l d B O E$ double mutant cells were starved on agar and allowed to develop for $24 \mathrm{~h}$. Bar, $0.5 \mathrm{~mm}$.

interaction between $p k c A$ and $p l d B$, and that PldB acts downstream of PkcA.

We also examined cell-substrate adhesion in $p k c A^{-}$, pldBOE, and $p k c A^{-} / p l d B O E$ cell lines (Fig. 3B). $p k c A^{-}$cells exhibited decreased cellsubstrate adhesion compared to wild-type cells, while both pldBOE and $p k c A^{-} / p l d B O E$ cells did not differ significantly from wild-type. This suggests that pldB overexpression can complement the defect caused by loss of $p k c A$, and restore normal cell-substrate adhesion. As was the case with cell-cell cohesion, this further suggests a functional interaction between $p k c A$ and $p l d B$, with PldB acting downstream of PkcA.

\section{6. pkcA effects are mediated through PLD activity}

Previous work has suggested that PldB mediates CMF signaling through the production of phosphatidic acid $[8,9]$. Our results suggest

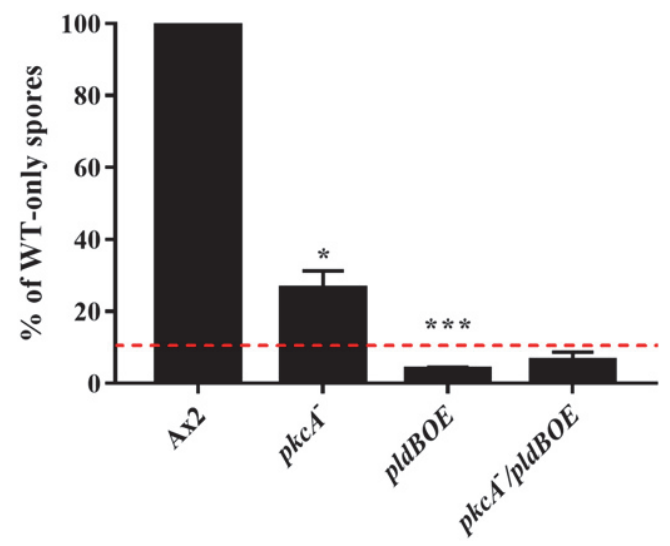

Fig. 2. $p k c A$ regulates spore formation. Chimeras comprised of $10 \% \mathrm{Ax} 2$, and $90 \%$ of the indicated mutant cell line were created and the total spores harvested. Total spore yield of chimera is expressed as a percentage of total spores collected from control fruiting bodies comprised of only Ax2 cells. Values are mean + SEM, from at least 3 independent experiments. Asterisk represents a significant difference from $10 \%$ wildtype spore yield, dashed red line $\left({ }^{*} \mathrm{P}<0.05 ;{ }^{* * *} \mathrm{P}<0.005\right)$, as determined by the $t$-test. that PkcA acts within the CMF signaling pathway, likely upstream of PldB. We have previously demonstrated that cells treated with exogenous phosphatidic acid behave as though PldB is overexpressed, reducing their ability to aggregate at low-densities, as though CMF signaling is blocked [8]. To determine whether PkcA acts through PldB within the CMF network, we modulated phosphatidic acid levels, to simulate changes in PLD-activity, and assessed their ability to aggregate (Table 2). To increase phosphatidic acid levels, we treated the cells with the cell permeable phosphatidic acid, 1,2-Dioctanoyl-sn-Glycero-3-Phosphate. Wild-type cells treated with phosphatidic acid were unable to aggregate below densities of $56 \times 10^{3}$ cells $/ \mathrm{cm}^{2}$, compared to $28 \times 10^{3}$ cells $/ \mathrm{cm}^{2}$ controls. $p k c A^{-}$cells treated with phosphatidic acid were unable to aggregate below $112 \times 10^{3}$ cells $/ \mathrm{cm}^{2}$, in stark contrast to their normal capability to aggregate down to a density of $7 \times 10^{3} \mathrm{cells} / \mathrm{cm}^{2}$. Addition of phosphatidic acid is capable of blocking aggregation in the $p k c A^{-}$cells, as though CMF signaling is turned off entirely.

To decrease intracellular phosphatidic acid levels, we treated the cells with 1-butanol. 1-Butanol functions as an inhibitor of PLD-activity by acting as an alternative, preferred substrate for the PLD-catalyzed transphosphatidylation reaction. This has the effect of reducing PLDderived PA in the cells, functionally behaving as though PLD has been inhibited [34]. Cells treated with butanol will aggregate at lower densities than the wild-type, as though CMF signaling is always active [9]. Wild-type cells treated with butanol were capable of aggregating down to $7 \times 10^{3}$ cells $/ \mathrm{cm}^{2}$, compared to untreated controls at $28 \times 10^{3}$ cells $/ \mathrm{cm}^{2}$. pkcAOE cells treated with butanol aggregated even at a density of $14 \times 10^{3} \mathrm{cells} / \mathrm{cm}^{2}$, whereas untreated cells were unable to aggregate at any density we tested. Addition of butanol restored aggregation in pkcAOE cells, suggesting that $\mathrm{CMF}$ signaling is turned on again. Taken together, these results suggest that PkcA acts within the CMF pathway, through PLD-mediated phosphatidic acid production.

\section{7. pkcA regulates PLD activity during development}

Our data suggests that PkcA functions upstream of PldB, possibly by regulating phosphatidic acid production by PldB. To determine if PkcA is capable of regulating PLD activity in D. discoideum, we measured PLD 

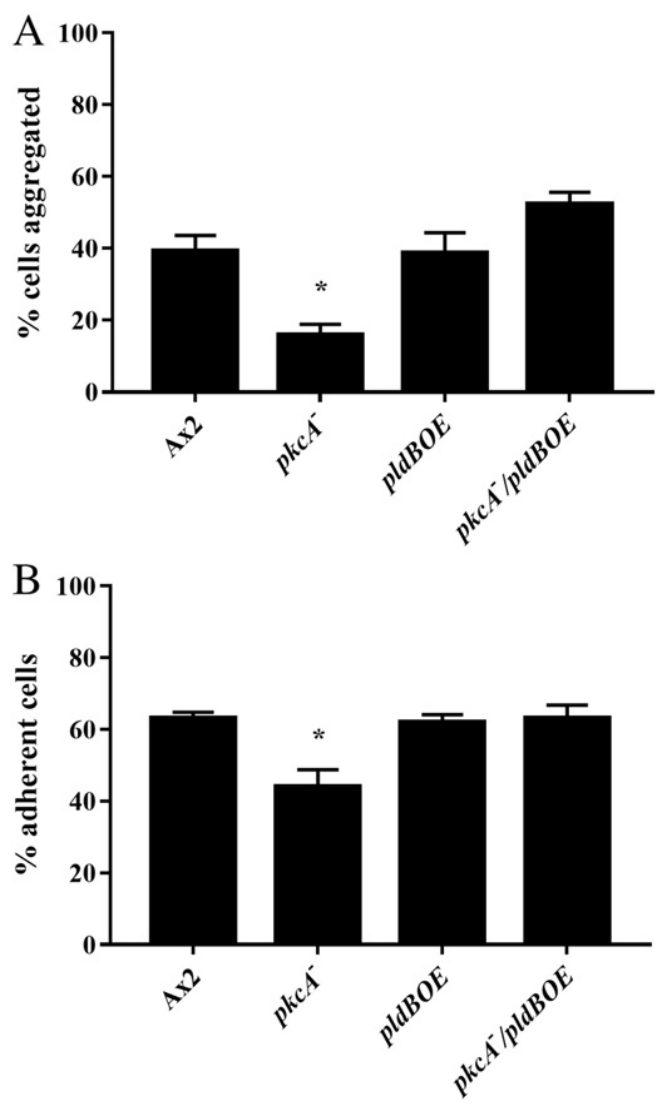

Fig. 3. pkcA regulates cell-cell cohesion and cell-substrate adhesion. (A) Cells were starved for $3 \mathrm{~h}$, aggregates were dissociated by vortexing, then allowed to re-adhere for $40 \mathrm{~min}$. Single/duplex cells were counted on a hemocytometer and the percentage of cells bound in aggregates calculated. (B) Cells were allowed to adhere to a glass bottomed flask for $2 \mathrm{~h}$. After gentle agitation for $5 \mathrm{~min}$, cells in the supernatant were counted on a hemocytometer and the percentage of adherent cells calculated. Values shown are mean + SEM, from at least 3 independent experiments. Asterisk represents a significant difference from wild-type $(P<0.005)$, as determined by the $t$-test.

activity in developing cells (Fig. 4). pkcA- cells exhibited a $~ 39 \%$ decrease in PLD activity compared to wild-type cells, while pkcAOE cells exhibited $\sim 60 \%$ increase in PLD activity compared to wild-type cells. These data show that knockout or overexpression of $p k c A$ is able to regulate intracellular PLD activity; at levels comparable to knockout or overexpression of pldB (Fig. 4).

\section{Table 2}

Effect of phosphatidic acid or butanol on low-cell density aggregation.

Cells were starved in PBM at the indicated cell densities in submerged monolayer culture with either $50 \mu \mathrm{M}$ 1, 2-dioctanoyl-sn-glycero-3-phosphate (PA) or $0.1 \%$ butanol. The field of cells was assessed for aggregation $(+)$ or non-aggregation $(-)$ after $24 \mathrm{~h}$, using an inverted microscope. Partial or substandard aggregation is represented as \pm (defined as where cells are migrating towards an aggregative center, but have not yet coalesced into an aggregate). Data shown are representative of at least 3 independent assays.

\begin{tabular}{|c|c|c|c|c|c|c|}
\hline \multirow[b]{2}{*}{ Cell type and treatment } & \multicolumn{6}{|c|}{$\begin{array}{l}\text { Presence of aggregates at cell density } \\
\left(10^{3} \text { cells } / \mathrm{cm}^{2}\right) \text { of: }\end{array}$} \\
\hline & 224 & 112 & 56 & 28 & 14 & 7 \\
\hline $\mathrm{Ax} 2$ & + & + & + & \pm & - & - \\
\hline $\mathrm{Ax} 2+50 \mu \mathrm{M}$ PA & + & + & \pm & - & - & - \\
\hline Ax $2+0.1 \%$ butanol & + & + & + & + & + & \pm \\
\hline$p k c A^{-}$ & + & + & + & + & + & \pm \\
\hline$p k c A^{-}+50 \mu \mathrm{M} \mathrm{PA}$ & + & + & - & - & - & - \\
\hline pkcAOE & - & - & - & - & - & - \\
\hline pkcAOE $+0.1 \%$ butanol & + & + & + & + & \pm & - \\
\hline
\end{tabular}

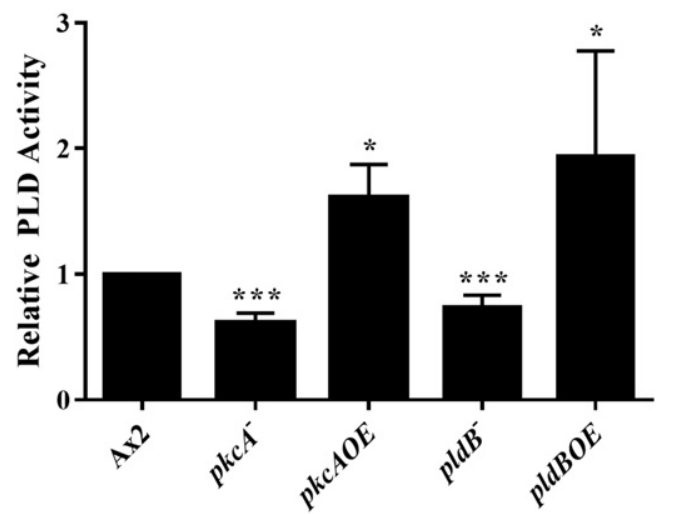

Fig. 4. $p k c A$ regulates PLD activity during development. Cells were starved for $6 \mathrm{~h}$ on filter pads, collected, lysed and the PLD activity measured. Values shown are mean + SEM, from at least 4 independent trials. Asterisk represents a significant difference from wild-type $\left({ }^{*} \mathrm{P}<0.05\right.$; $\left.{ }^{* * *} \mathrm{P}<0.005\right)$, as determined by the $t$-test.

\section{8. pkcA regulates cell polarity, but not filopodia formation}

Improper organization of the actin cytoskeleton can cause developmental defects, partly due to altered chemotaxis and motility [35-38]. Cells lacking PkcA have increased actin polymerization rates in response to cAMP, as well as visibly altered cell morphology during chemotaxis [14]. To investigate the role of $p k c A$ in regulating cell shape, we starved the cells on a coverslip for $6 \mathrm{~h}$, then stained the actin cytoskeleton (Fig. 5). We measured the Roundness of the cells, as a measure of their ability to polarize and elongate along the axis of the cAMP gradient, which
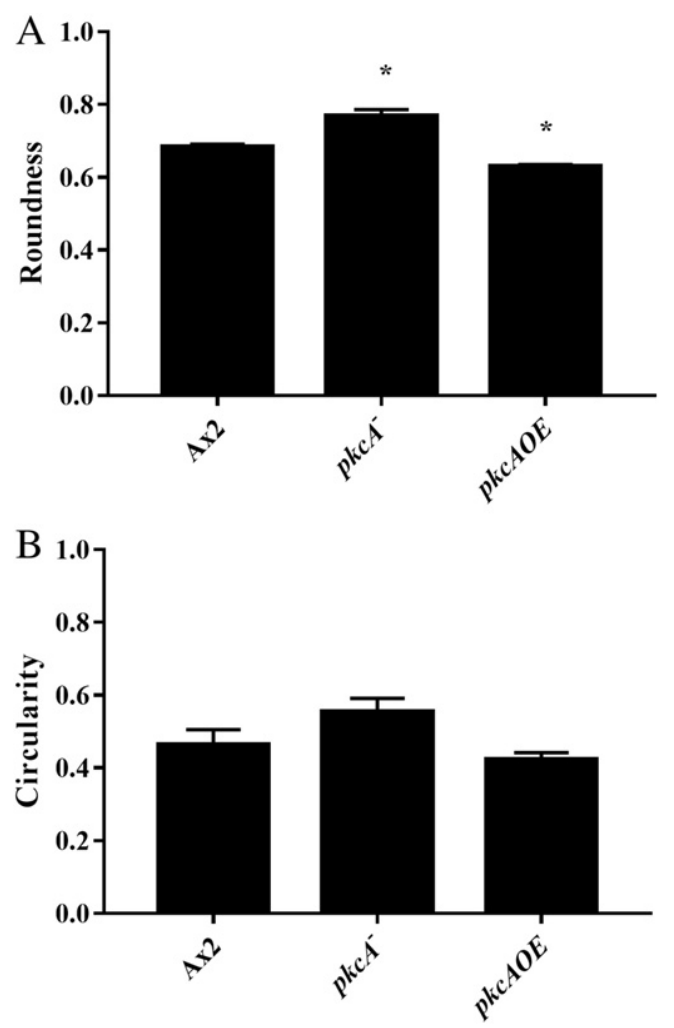

Fig. 5. $p k c$ A regulates cell polarity, does not regulate filopodia formation. Cells were starved for $6 \mathrm{~h}$ on a coverslip, then fixed and stained to visualize the actin cytoskeleton. (A) Roundness (minor axis/major axis) or (B) Circularity (4m[area] $/$ perimeter $^{2}$ ) were calculated using ImageJ software. Values are means of at least 3 independent trials + SEM, in which at least 100 cells were evaluated per trial. Asterisk represents a significant difference from wild-type $(P<0.05)$, as determined by the $t$-test. 
involves the suppression of lateral pseudopod formation [36]. We also measured the Circularity of the cells, as a measure of the cells' ability to form filopodia along its periphery, which involves organizing Factin to create these structures. Roundness is defined as the ratio of minor axis of the cell to the major axis, length of minor axis length of major axis. $A$ cell that is perfectly round will have a Roundness of 1 , which decreases as the cell elongates. We found that $p k c A^{-}$cells had increased Roundness compared to wild-type, while $p k c A O E$ cells exhibited decreased Roundness compared to wild-type. Our data suggest PkcA may regulate cell shape either by suppressing lateral pseudopods during chemotaxis, or by regulating forward pseudopod generation at the anterior of the cell. Circularity is defined as $\frac{4 \pi(\text { area })}{\text { perimeter }^{2}}$. A cell that is shaped like a perfect circle will have a Circularity measure of 1 , which decreases as the cell produces filopodia. This is due to an increased overall cell membrane perimeter, with minimal change in the overall cell surface area. We found that neither mutant cell line exhibited a significant change in Circularity from the wild-type when starved. PkcA does not seem to regulate the formation of filopodia.

\section{9. pkcA regulates nuclear segregation}

In addition to regulating cytoskeletal organization, Protein Kinase C isoforms are regulators of nuclear segregation $[21,39,40]$. To determine whether $p k c A$ plays a role during nuclear segregation, we looked at the multinuclearity state of populations of vegetative cells (Fig. 6). Log phase cells were allowed to adhere to a coverslip, then stained with DAPI and the number of nuclei/cell determined. We found that $p k c A^{-}$ cells had a significant fraction of cells containing 2, or 3 or more nuclei, when compared to wild-type cells. The population of single or multinucleate pldBOE cells did not differ significantly from those of wild-type. These data suggest that $p k c A$, but not $p l d B$, plays a role in regulating nuclear segregation. Overexpression of pldB is capable of complementing the $p k c A^{-}$defect, restoring the population of multinucleate cells back to wild-type proportions. This suggests that proper nuclear segregation may involve PLD, whose activity is decreased in $p k c A^{-}$cells, but complemented by overexpression of PldB.

\section{Discussion}

The initiation of aggregation in $D$. discoideum is triggered by the accumulation of $\mathrm{CMF}$, an extracellular signaling protein produced by starving cells [41,42]. CMF accumulation leads to GPCR-mediated activation of phospholipase C, which produces diacylglycerol [11].

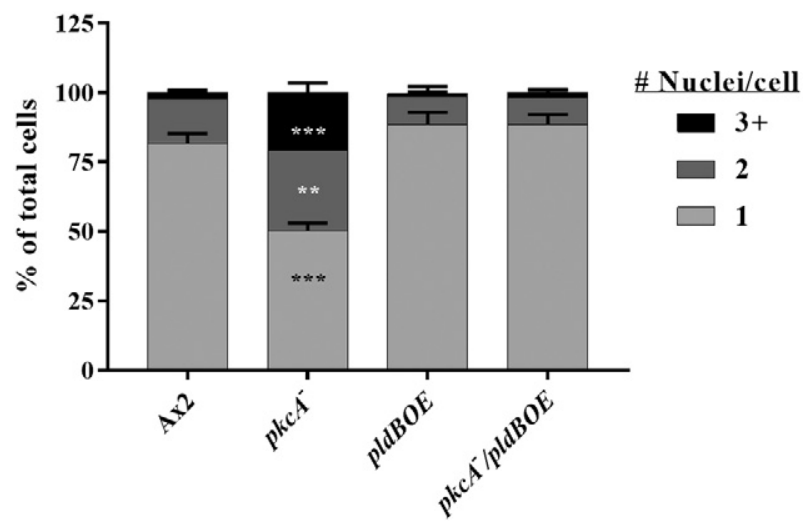

Fig. 6. $p k c A$ regulates nuclear segregation. Cells were allowed to settle on a coverslip for $1 \mathrm{~h}$, fixed and the nuclei stained. Cells were imaged by both brightfield and epifluorescence microscopy, and the number of nuclei/cell were calculated. Values shown are means of at least 3 independent trials + SEM, in which at least 150 cells were evaluated per trial. Asterisks represent a significant difference from wild-type $\left.{ }^{* *} P<0.01 ;{ }^{* * *} P<0.005\right)$ as determined by the $t$-test.
Activation of phospholipase $\mathrm{C}$ has been associated with the downregulation of PldB, which allows initiation of development [8]. Until now, no link between PLC and PIdB has been established and we propose that PkcA may act in between these two proteins.

Here we show that PkcA acts within the quorum sensing pathway to regulate entry into development. Cells lacking PkcA are capable of aggregating down to very low densities, as though their quorum sensing pathway is always active. Conversely, cells overexpressing PkcA do not aggregate at any density, as though their quorum sensing is blocked, rendering them incapable of aggregating or progressing through development. This suggests that PkcA is critical to quorum sensing, and is a negative regulator of CMF signaling, much like PldB. This is supported by the similarities of the $p k c A^{-}$to $p l d B^{-}$, which is thought to link the $\mathrm{CMF}$ /quorum sensing signaling pathway to the cAR1 signaling response [8].

The similarities in the aggregative phenotypes of the $p k c A$ mutants to those of the pldB mutants suggest that they functionally interact to regulate aggregation, and possibly other developmentally relevant processes. Here, we utilize epistasis analysis using the $p k \mathrm{cA}^{-} / \mathrm{pldBOE}$ double mutant to determine whether PkcA and PldB function within the same pathway(s), and if so, to determine their order of activity within those pathways. For a given pathway, the phenotype of the double mutant will phenocopy the parental phenotype of the protein that acts downstream. For example, the $p k c A^{-}$cells are able to aggregate at low densities, down to $7 \times 10^{3}$ cells $/ \mathrm{cm}^{2}$. Conversely, the pldBOE cells will not aggregate at any tested density. The $p k c A^{-} / p l d B O E$ cells are also incapable of aggregating at any tested density, phenocopying the pldBOE parent cell line. Epistasis analysis concludes that PldB acts downstream of PkcA during aggregation. This relationship appears to hold true for fruiting body formation, spore formation, cell-cell cohesion, and cellsubstrate adhesion. In addition, $p k c A^{-}$and $p l d B^{-}$cells share common phenotypes, arguing that PkcA is a positive regulator of PldB.

PkcA may regulate PldB by controlling its enzymatic activity. As a PLD, PldB cleaves phosphatidylcholine to produce choline and phosphatidic acid $[8,9]$. It has previously been demonstrated that PldB's signaling activity is mediated through phosphatidic acid production [8]. Here, we show that PkcA's activity is also mediated through phosphatidic acid production during aggregation. We also show that PkcA is able to regulate total phosphatidic acid production in Dictyostelium cells during development. Loss of PkcA caused a decrease in phosphatidic acid levels similar to that seen in cells lacking PldB. D. discoideum possesses three orthologues of the mammalian PLD1, pldA, pldB, and pldC. Of these three, only PldB has a developmental function similar to that of PkcA $[8,9]$. Taken together, this supports the idea that PkcA serves as an activator of PldB.

PkcA appears to serve as a positive regulator of PldB, with both proteins having a negative regulatory role in aggregation. Given that PldB activity must be downregulated for proper aggregation, and that this is likely mediated by PLC-activity during development [5,8], there may be another DAG-responsive protein(s) that serves as a negative regulator of PldB. In mammalian cells, PKC can both activate and inactivate PLD, through binding interactions and/or phosphorylation [27]. Our results suggest PkcA activates PldB, although the mechanism needs to be determined. Another PKC-class protein may serve the function of inactivating PldB. One potential candidate protein is PakD. Like PkcA, PakD is a putative DAG-responsive Ser/Thr-kinase which is expressed during the first 8-h of development, the same time frame as both PkcA and PldB $[9,14,43]$. Strikingly, the roles of PakD are precisely opposed to the roles of PkcA and PldB. Compared to PkcA and PldB, which are negative regulators, PakD serves as a positive regulator of aggregation and development [43]. These strongly opposed phenotypes suggest that PkcA and PakD may serve opposing functions during the regulation of aggregation and chemotaxis (Fig. 7). It is possible that PakD may inactivate either PkcA or PldB, allowing aggregation to occur, although no direct links have been established yet between PakD and either PkcA or PldB. 


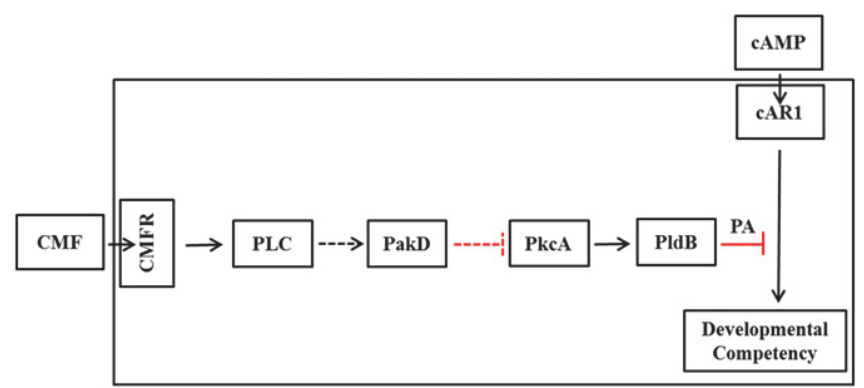

Fig. 7. Proposed model of CMF signal transduction. At the onset of starvation, cAMP signaling through cAR1 is blocked by PldB-mediated phosphatidic acid (PA) generation, which is regulated by PkcA. When sufficient cells begin to starve, CMF binding to its receptor induces the downstream activation of PLC. Activated PLC may activate PakD, which may serve to inactivate PkcA. The downregulation of PkcA results in a corresponding reduction of PldB-activity, and phosphatidic acid levels. This allows for cAR1-mediated cAMP signaling to occur, allowing for aggregation and development to proceed.

We also find that $p k c A^{-}$mutant cells have decreased polarization when chemotaxing towards cAMP. Conversely, cells overexpressing PkcA have increased polarity when chemotaxing to cAMP. This suggests that PkcA may play a role in sensing the direction of a cAMP source, and organizing the cytoskeleton to polarize in response. $p k c A^{-}$cells have altered actin polymerization in response to cAMP stimulation [14]. Wildtype cells exhibit two peaks of F-actin polymerization when stimulated with cAMP. The first is at $5 \mathrm{~s}$, corresponding to a rapid burst of F-actin polymerization throughout the cortex, after which levels of F-actin drop substantially. The second, lesser peak is at 30-60 s after stimulation, and begins to diminish after $\sim 80 \mathrm{~s}$. This slower response corresponds to the extension of lateral pseudopods, that helps the cells orient towards the strongest source of cAMP in the environment [44]. $p k c A^{-}$cells have significantly higher levels of F-actin at $5 \mathrm{~s}$, which slowly decays over time down to a slightly elevated level of F-actin over the basal level by $\sim 30-40 \mathrm{~s}$, eliminating the second peak. The loss of the second F-actin polymerization peak at $30 \mathrm{~s}$ could lead to diminished ability of the cells to extend pseudopods and orient towards the source of cAMP. When combined with increased cortical stiffness due to elevated actin content in the cortex [45], this could lead to a decrease in the cells' ability to polarize in response to cAMP.

Finally, we describe a potential function of PkcA during vegetative growth. It is important to note that while $p k c A$ expression has only been demonstrated in developing cells, this does not rule out a basal level of expression in vegetative cells below detection thresholds. In cells lacking PkcA, a significant proportion of cells contain an abnormal number of nuclei compared to wild-type cells. This could suggest that PkcA may play a role in segregating the nuclei between dividing daughter cells. There is evidence from both humans and yeast that PKC can interact with topoisomerase II and may play a role in mitotic spindle formation, chromosome segregation, and regulation of the G2-M transition $[39,40,46]$. Interestingly, overexpression of PldB in vegetative cells is capable of complementing the multinuclearity defect of $p k c A^{-}$cells. PldB overexpression restores the average nuclei/cell proportions back to wild-type levels. Phosphatidic acid produced by PLD is known to play a role in regulation of the cell cycle and cell division $[47,48]$. There are vegetatively expressed PLD orthologues in $D$. discoideum [49], which may be regulated by PkcA during growth, in turn regulating cell division. While PldB is only produced during development, vegetative overexpression, and the resultant production of phosphatidic acid, may compensate for disrupted signaling of the vegetatively expressed phospholipases due to loss of PkcA.

We show that PkcA serves as a negative regulator of aggregation and development, as well as a regulator of cell shape in Dictyostelium discoideum. Additionally, we have established a functional relationship between PkcA and PldB, wherein they cooperatively regulate cells' entry into the developmental program, as well as aggregation, cell differentiation, and culmination. While no direct link between PkcA and PIdB has been demonstrated, the presence of a PLC orthologue involved in regulation of quorum sensing may provide clues to how they interact. Phospholipase $C$ produces diacylglycerol upon activation [50]. DAG may induce translocation of PkcA to the plasma membrane, putting it in close proximity to PldB, which is known to associate with the actin cortex through the adaptor protein PaxB [10]. PkcA and PldB may interact at the membrane, with PkcA regulating PldB-mediated production of phosphatidic acid, in turn regulating the progression of development.

\section{Conclusions}

- PkcA regulates the initiation of development through the CMF-quorum sensing pathway, serving as a negative regulator of aggregation.

- PkcA regulates cell shape, and the organization of the actin cytoskeleton in response to cAMP.

- Epistasis analysis shows that PkcA and PldB cooperatively interact to regulate a number of developmentally critical processes, including adhesion, cell sorting, and differentiation. PkcA likely acts upstream of PldB.

- PkcA regulates the PLD-activity of PldB, indirectly serving as a negative regulator of many developmentally relevant processes.

- PkcA may regulate nuclear segregation during mitosis, in vegetative cells.

\section{Acknowledgements}

We would like to thank Dr. Sibnath Ray for the creation of the $p k c A$ mutant strains, as well as Dr. Rebecca Garcia for training and assistance of the students involved. This work was supported by grants to D.B. from the National Science Foundation (MCB-1244162), a PSC-CUNY grant (69271 00 47), as well as Research Centers in Minority Institutions Program grants from the National Institute on Minority Health and Health Disparities (8 G12 MD007599) from the National Institutes of Health.

\section{References}

[1] J.D. Gross, Dictyostelium discoideum: A Developmental System, Elsevier, 1975, http:// dx.doi.org/10.1016/0092-8674(75)90048-3.

[2] R.H. Kessin, Dictyostelium: Evolution, Cell Biology, and the Development of Multicellularity, Cambridge University Press, 2001.

[3] L. Strmecki, D.M. Greene, C.J. Pears, Developmental decisions in Dictyostelium discoideum, Dev. Biol. 284 (2005) 25-36, http://dx.doi.org/10.1016/j.ydbio.2005. 05.011 .

[4] W.F. Loomis, Cell signaling during development of dictyostelium, Dev. Biol. 391 (2014) 1-16, http://dx.doi.org/10.1016/j.ydbio.2014.04.001.

[5] R.H. Gomer, W. Jang, D. Brazill, Cell density sensing and size determination, Develop. Growth Differ. 53 (2011) 482-494, http://dx.doi.org/10.1111/j.1440-169X.2010. 01248.x.

[6] I.S. Yuen, R.H. Gomer, Cell density-sensing in Dictyostelium by means of the accumulation rate, diffusion coefficient and activity threshold of a protein secreted by starved cells, J. Theor. Biol. 167 (1994) 273-282, http://dx.doi.org/10.1006/jtbi. 1994.1069.

[7] R.H. Gomer, I.S. Yuen, R.A. Firtel, A secreted $80 \times 10$ (3) Mr protein mediates sensing of cell density and the onset of development in Dictyostelium, Development 112 (1991) 269-278 http://www.ncbi.nlm.nih.gov/pubmed/1663029 (accessed September 9 , 2015).

[8] S. Ray, Y. Chen, J. Ayoung, R. Hanna, D. Brazill, Phospholipase D controls Dictyostelium development by regulating G protein signaling, Cell. Signal. 23 (2011) 335-343, http://dx.doi.org/10.1016/j.cellsig.2010.09.017.

[9] Y. Chen, V. Rodrick, Y. Yan, D. Brazill, PldB, a putative phospholipase D homologue in Dictyostelium discoideum mediates quorum sensing during development, Eukaryot. Cell 4 (2005) 694-702, http://dx.doi.org/10.1128/EC.4.4.694-702.2005.

[10] J. Pribic, R. Garcia, M. Kong, D. Brazill, Paxillin and phospholipase D interact to regulate actin-based processes in Dictyostelium discoideum, Eukaryot. Cell 10 (2011) 977-984, http://dx.doi.org/10.1128/EC.00282-10.

[11] D.T. Brazill, D.F. Lindsey, J.D. Bishop, R.H. Gomer, Cell density sensing mediated by a G protein-coupled receptor activating phospholipase C, J. Biol. Chem. 273 (1998) 8161-8168, http://dx.doi.org/10.1074/jbc.273.14.8161.

[12] M.J. Rebecchi, S.N. Pentyala, Structure, function, and control of phosphoinositidespecific phospholipase C, Physiol. Rev. 80 (2000) 1291-1335 (doi:0031-9333/00). 
[13] A.B. Cubitt, S. Dharmawardhane, R.A. Firtel, Developmentally regulated changes in 1,2-diacylglycerol in Dictyostelium. Regulation by light and G proteins, J. Biol. Chem. 268 (1993) 17431-17439 http://www.ncbi.nlm.nih.gov/pubmed/8394342 (accessed October 4, 2016).

[14] W. Mohamed, S. Ray, D. Brazill, R. Baskar, Absence of catalytic domain in a putative protein kinase C (PkcA) suppresses tip dominance in Dictyostelium discoideum, Dev. Biol. 405 (2015) 10-20, http://dx.doi.org/10.1016/j.ydbio.2015.05.021.

[15] S.F. Steinberg, Structural basis of protein kinase $C$ isoform function, Physiol. Rev. 88 (2008) 1341-1378, http://dx.doi.org/10.1152/physrev.00034.2007.

[16] S. Ni, L. Chen, M. Li, W. Zhao, X. Shan, M. Wu, J. Cheng, L. Liang, Y. Wang, W. Jiang, J. Zhang, R. Ni, PKC iota promotes cellular proliferation by accelerated G1/S transition via interaction with CDK7 in esophageal squamous cell carcinoma, Tumor Biol. 1-11 (2016), http://dx.doi.org/10.1007/s13277-016-5193-9.

[17] A.C. Newton, Protein kinase C: poised to signal, Am. J. Physiol. Endocrinol. Metab. 298 (2010) E395-E402, http://dx.doi.org/10.1152/ajpendo.00477.2009.

[18] L.B. Luna-Ulloa, J.G. Hernández-Maqueda, M.C. Castañeda-Patlán, M. Robles-Flores, Protein kinase $C$ in Wnt signaling: implications in cancer initiation and progression, IUBMB Life 63 (2011) 873-879, http://dx.doi.org/10.1002/iub.559.

[19] H. Xiao, M. Liu, Atypical protein kinase C in cell motility, Cell. Mol. Life Sci. 70 (2013) 3057-3066, http://dx.doi.org/10.1007/s00018-012-1192-1.

[20] X. Feng, J. Zhang, K. Smuga-Otto, S. Tian, J. Yu, R. Stewart, J.A. Thomson, Protein kinase $C$ mediated extraembryonic endoderm differentiation of human embryonic stem cells, Stem Cells 30 (2012) 461-470, http://dx.doi.org/10.1002/stem.1018.

[21] B.S. Fogh, H.A. Multhaupt, J.R. Couchman, Protein kinase C, focal adhesions and the regulation of cell migration, J. Histochem. Cytochem. 62 (2014) 172-184, http:// dx.doi.org/10.1369/0022155413517701.

[22] C. Cataisson, A.J. Pearson, S. Torgerson, S.A. Nedospasov, S.H. Yuspa, Protein kinase C-mediated chemotaxis of neutrophils requires NF-B activity but is independent of TNF signaling in mouse skin in vivo, J. Immunol. 174 (2005) 1686-1692, http:// dx.doi.org/10.4049/jimmunol.174.3.1686.

[23] I. Michalczyk, A. Sikorski, The emerging role of protein kinase $\mathrm{C} \theta$ in cytoskeletal signaling, J. Leukoc. 93 (2013) 319-327, http://dx.doi.org/10.1189/jlb.0812371.

[24] Q. Yang, X.-F. Zhang, D. Van Goor, A.P. Dunn, C. Hyland, N. Medeiros, P. Forscher, Protein kinase $C$ activation decreases peripheral actin network density and increases central nonmuscle myosin II contractility in neuronal growth cones, Mol. Biol. Cell 24 (2013) 3097-3114, http://dx.doi.org/10.1091/mbc.E13-05-0289.

[25] D. Breitkreutz, L. Braiman-Wiksman, N. Daum, M.F. Denning, T. Tennenbaum, Protein kinase C family: on the crossroads of cell signaling in skin and tumor epithelium, J. Cancer Res. Clin. Oncol. 133 (2007) 793-808, http://dx.doi.org/10.1007/ s00432-007-0280-3.

[26] L.L. Rodriguez, I.C. Schneider, Directed cell migration in multi-cue environments, Integr. Biol. 5 (2013) 1306-1323, http://dx.doi.org/10.1039/c3ib40137e.

[27] T. Hu, J.H. Exton, Mechanisms of regulation of phospholipase D1 by protein kinase Ca, J. Biol. Chem. 278 (2003) 2348-2355, http://dx.doi.org/10.1074/jbc. M210093200.

[28] X. Xu, T. Jin, The novel functions of the PLC/PKC/PKD signaling axis in G proteincoupled receptor-mediated chemotaxis of neutrophils, J. Immunol. Res. 2015 (2015) 14-19, http://dx.doi.org/10.1155/2015/817604.

[29] K.P. Becker, Y.A. Hannun, Protein kinase $C$ and phospholipase D: intimate interactions in intracellular signaling, Cell. Mol. Life Sci. 62 (2005) 1448-1461, http://dx. doi.org/10.1007/s00018-005-4531-7.

[30] F. Pủta, C. Zeng, Blasticidin resistance cassette in symmetrical polylinkers for insertional inactivation of genes in Dictyostelium, Folia Biol. (Praha). 44 (1998) 185-188 http://www.ncbi.nlm.nih.gov/pubmed/10732710 (accessed November 14, 2016).

[31] K.M. Pang, M. a Lynes, D. a Knecht, Variables controlling the expression level of exogenous genes in Dictyostelium, Plasmid 41 (1999) 187-197, http://dx.doi.org/10. 1006/plas.1999.1391.
[32] R. Jain, R.H. Gomer, A developmentally regulated cell surface receptor for a densitysensing factor in Dictyostelium, J. Biol. Chem. 269 (1994) 9128-9136 http://www. jbc.org/content/269/12/9128 (accessed August 20, 2015).

[33] M.B. Duran, A. Rahman, M. Colten, D. Brazill, Dictyostelium discoideum paxillin regulates actin-based processes, Protist 160 (2009) 221-232, http://dx.doi.org/10.1016/ j.protis.2008.09.005.

[34] Y. Shen, L. Xu, D.A. Foster, Role for phospholipase D in receptor-mediated endocytosis, Mol. Cell. Biol. 21 (2001) 595-602, http://dx.doi.org/10.1128/MCB.21.2.595-602. 2001.

[35] P. Devreotes, A.R. Horwitz, Signaling networks that regulate cell migration, Cold Spring Harb. Perspect. Biol. 7 (2015) a005959, http://dx.doi.org/10.1101/ cshperspect.a005959.

[36] H.P. Williams, A.J. Harwood, Cell polarity and Dictyostelium development, Curr. Opin. Microbiol. 6 (2003) 621-627, http://dx.doi.org/10.1016/j.mib.2003.10.008.

[37] L. Stephens, L. Milne, P. Hawkins, Moving towards a better understanding of chemotaxis, Curr. Biol. 18 (2008) 485-494, http://dx.doi.org/10.1016/j.cub.2008.04.048.

[38] Y. Artemenko, T.J. Lampert, P.N. Devreotes, Moving towards a paradigm: common mechanisms of chemotactic signaling in Dictyostelium and mammalian leukocytes, Cell. Mol. Life Sci. 71 (2014) 3711-3747, http://dx.doi.org/10.1007/s00018-0141638-8.

[39] C.R. Barker, N.A.P. Mouchel, J.R. Jenkins, The identification and characterisation of a functional interaction between arginyl-tRNA-protein transferase and topoisomerase II, Biochem. Biophys. Res. Commun. 342 (2006) 596-604, http://dx.doi.org/10.1016/ j.bbrc.2006.02.006.

[40] A.M. Fry, Cell cycle phase-specific phosphorylation of human topoisomerase Ilalpha, J. Biol. Chem. 270 (1995) 28357-28363, http://dx.doi.org/10.1074/jbc.270.47.28357.

[41] W.J. Deery, T. Gao, R. Ammann, R.H. Gomer, A single cell density-sensing factor stimulates distinct signal transduction pathways through two different receptors, J. Biol. Chem. 277 (2002) 31972-31979, http://dx.doi.org/10.1074/jbc.M204539200.

[42] D.T. Brazill, R. Gundersen, R.H. Gomer, A cell-density sensing factor regulates the lifetime of a chemoattractant-induced G??-GTP conformation, FEBS Lett. 404 (1997) 100-104, http://dx.doi.org/10.1016/S0014-5793(97)00104-X.

[43] M. Garcia, S. Ray, I. Brown, J. Irom, D. Brazill, PakD, a putative p21-activated protein kinase in Dictyostelium discoideum, regulates actin, Eukaryot. Cell 13 (2014) 119-126, http://dx.doi.org/10.1128/EC.00216-13.

[44] L. Chen, Two phases of actin polymerization display different dependencies on $\mathrm{PI}(3,4,5) \mathrm{P} 3$ accumulation and have unique roles during chemotaxis, Mol. Biol. Cell 14 (2003) 5028-5037, http://dx.doi.org/10.1091/mbc.E03-05-0339.

[45] Y. Shimizu, S.M.A. Haghparast, T. Kihara, J. Miyake, Cortical rigidity of round cells in mitotic phase and suspended state, Micron 43 (2012) 1246-1251, http://dx.doi.org/ 10.1016/j.micron.2012.03.011.

[46] D.E. Levin, F.O. Fields, R. Kunisawa, J.M. Bishop, J. Thorner, A candidate protein kinase C gene, PKC1, is required for the S. cerevisiae cell cycle, Cell 62 (1990) 213-224, http://dx.doi.org/10.1016/0092-8674(90)90360-Q.

[47] C.S. Lee, K.L. Kim, J.H. Jang, Y.S. Choi, P.G. Suh, S.H. Ryu, The roles of phospholipase D in EGFR signaling, Biochim. Biophys. Acta Mol. Cell Biol. Lipids 1791 (2009) 862-868, http://dx.doi.org/10.1016/j.bbalip.2009.04.007.

[48] D. a Foster, L. Xu, Phospholipase D in cell proliferation and cancer, Mol. Cancer Res. 1 (2003) 789-800 http://www.ncbi.nlm.nih.gov/pubmed/14517341 (accessed October 5,2016$)$

[49] S. Zouwail, T.R. Pettitt, S.K. Dove, M.V. Chibalina, D.J. Powner, L. Haynes, M.J.O Wakelam, R.H. Insall, Phospholipase D activity is essential for actin localization and actin-based motility in Dictyostelium, Biochem. J. 389 (2005) 207-214, http://dx.doi.org/10.1042/BJ20050085.

[50] a Shariff, E.J. Luna, Diacylglycerol-stimulated formation of actin nucleation sites at plasma membranes, Science 256 (1992) 245-247, http://www.ncbi.nlm.nih.gov/ pubmed/1373523. 